\title{
Reliability Analysis of MIPS Reconfigurable Embedded System
}

\author{
Jinfeng $\mathrm{Li}^{1, \mathrm{a}^{*}}$, Wenjie Zhang ${ }^{1, \mathrm{~b}}$ and Renbo $\mathrm{Xu}^{1,2, \mathrm{c}}$ \\ ${ }^{1}$ Nanchang Institute of Science and Technology, Nanchang, 330108, China \\ ${ }^{2}$ Physics and Microelectronics Institute, Central South University Changsha 410083 china. \\ a524892454@qq.com, b568740355@qq.com, ${ }^{c} 1310079831 @ q q . c o m$
}

Keywords: MIPS; Dynamic reconfiguration; Markov process; Reliability

\begin{abstract}
Dynamic reconstruction technology has been widely used in the field of embedded systems. Because of the limited chip resources, multiple processors are embedded at the same time, which takes up too much chip space. So, In order to save limited chip space, developers often use dynamic refactoring techniques in multiprocessor technology. Refactoring technology has the functions of solving the problems of limited resource, improving system reliability and reducing system power consumption. Reconstruct processor of this paper studies is the MIPS micro processing unit, including the three model reconstruction and four model reconstruction pattern. We then use Markov processes to study their reliability, respectively. At present, there is little research in this field in our country, so it is very meaningful to analyze the reliability of Reconfigurable Embedded system.
\end{abstract}

\section{Introduction}

The reconfigurable operation of field programmable gate array (FPGA) is divided into dynamic reconfiguration and static reconstruction[1]. Dynamic reconfiguration has the ability to refactor circuit logic when the system is running. Static reconfiguration requires the system to stop operating when the circuit logic is reconstructed[2]. The advantage of reconfigurable computing is that it has the flexibility of software, the variable architecture and the execution speed of traditional hardware design to a certain extent. It is well adapted to the diversified needs of practical applications[3].

Markov process is a kind of stochastic process. It was first proposed by Russian mathematician Markov in 1907[4]. The characteristics of the process are as follows: the evolution of the next state does not depend on the evolution of the previous state under the condition of known conditions. In reality, many process is a Markov process, such as the Brown movement of fine particles in the liquid; the number of vehicles of crossroads; the number of infectious disease infected; the number of students in the classroom through the door; the station waiting number, can be regarded as a Markov process[5]. With the study of Markov processes, many scientists have begun to study and apply the process in nineteenth Century, which lays the theoretical foundation for Markov process[6].

\section{Overall Structure of the Third Mock Redundant System}

This paper studies the third mock redundancy system of Reconfigurable Embedded System for MIPS, unit and equipment are designed are: three MIPS micro processing unit; instruction storage unit; a data storage unit; comparator; the latch; two LED lamp; a switch; a host; a download cable and FPGA development board. As shown in Fig.1, the dotted line frame is a reconfigurable module, and the solid line frame is a sub module. 


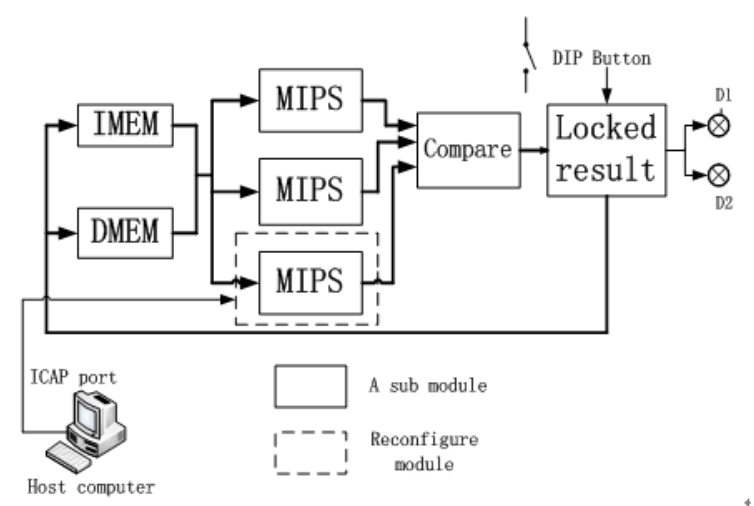

Figure 1. MIPS redundant structure of the third mock mode

\section{The Reliability Analysis of the Third Mock System Redundancy}

This paper uses Markov process to analyze MIPS reliability of the third mock for Reconfigurable Embedded system.

Authors and Affiliations Homogeneous Markov Process. Let the random process $\{\mathrm{X}(\mathrm{T}), \mathrm{t}$, $\mathrm{T}\}$, in which time is $\mathrm{T}=\{0,1,2,3, \ldots\}$, the status space is $\mathrm{I}=\{0,1,2,3, \ldots\}$, if at any time, $\mathrm{N}$, and any state, $i$ and $j$, have $P\{X(n+1)=j \mid X(n)=i, X(n-1)=i n-1, \ldots X(1)=i 1, X(0)=i 0\}=P\{X(n+1),=j$ $\mid X(n)=i\}$, then $\{X(T), t T\}$ random process is a Markov chain, that is, $\{X n, n>0\}$. This shows that the probability distribution of the $X(n)$ at the moment $n+1$ is in the state $j$ in the stochastic process, and is only related to the state $\mathrm{i}$ of the $\mathrm{n}$ moment, but with the previous state in-1, ... I1, I0 independent, such a process is called a Markov process[7].

The so-called homogeneous Markov process means that for any state, $\mathrm{i}$ and $\mathrm{j}$, there are formulas (1) established.

$$
P\{\mathrm{X}(\mathrm{t}+\Delta \mathrm{t})=\mathrm{j} \mid \mathrm{X}(\mathrm{t})=\mathrm{i}\}=P\{\mathrm{X}(\Delta \mathrm{t})=\mathrm{j} \mid \mathrm{X}(0)=\mathrm{i}\}=P_{\mathrm{ij}}(\Delta \mathrm{t})
$$

By formula (1), the transition from the state $\mathrm{i}$ to the state $\mathrm{j}$ is only related to the time difference between $\Delta \mathrm{T}$, and it is independent of the starting time t. Pij $(\Delta \mathrm{T})$ is the transition probability of the state $\mathrm{i}$ to the state $\mathrm{j}$, then the matrix consisting of Pij (delta $\mathrm{T}$ ) is called the transfer matrix [8]. The state transition matrix is defined as a formula (2):

$$
p(\Delta \mathrm{t})=\left[\begin{array}{cccc}
P 11(\Delta \mathrm{t}) & P 12(\Delta \mathrm{t}) & \ldots & P n 1(\Delta \mathrm{t}) \\
P 21(\Delta \mathrm{t}) & P 22(\Delta \mathrm{t}) & \ldots & P n 2(\Delta \mathrm{t}) \\
\ldots & \ldots & \ldots & \ldots \\
P n 1(\Delta \mathrm{t}) & P n 2(h) & \ldots & P n n(\Delta \mathrm{t})
\end{array}\right]
$$

Define the transfer density matrix $Q$, such as formula (3):

$$
Q=\lim _{h \rightarrow 0}(\mathrm{p}(\Delta \mathrm{t})-\mathrm{I}) / \Delta \mathrm{t}=\left[\begin{array}{cccc}
q 11 & q 12 & \ldots & q n 1 \\
q 21 & q 22 & \ldots & q n 2 \\
\ldots & \ldots & \ldots & \ldots \\
q n 1 & q n 2 & \ldots & q n n
\end{array}\right]
$$

From formula (2) and formula (3) can be obtained : ${ }^{\frac{\mathrm{d}}{\mathrm{d}(\mathrm{t})} p(t)=p(\mathrm{t}) Q}$, among them, I is the unit matrix, $\mathrm{P}(\mathrm{t}),=\{\mathrm{p} 1(\mathrm{t}), \mathrm{P} 2(\mathrm{t}), \ldots\}$. At any time $\mathrm{t}$, according to $\mathrm{P}(\mathrm{t})$, the probabilities of each state can be obtained. 
Reliability Analysis. In terms of structure and function, the three MIPS micro processing units to be reconstructed are exactly the same, and the three MIPS modules are isomorphic to each other. The operation of the system can be divided into 7 states.

Status 0: the initial state, the three modules are no fault, the system works properly. Status 6: three modules have failed, and three modules output results are different, at this time the system can not get the correct results.

Assume that a module can work normally at the moment $t$, the probability of failure in the $t+\Delta t$ is $\mathrm{p}=\lambda \Delta \mathrm{t}$ ( $\lambda$ as the failure rate), $\mathrm{C}$ for fault detection coverage probability (probability of any fault processing module is detected), then processing fault probability and detected is $\mathrm{C} \lambda \Delta \mathrm{t}$, the probability can not be detected is (1-C) $\lambda \Delta \mathrm{t}$. If a module fails at the time $\mathrm{t}$, the probability of being repaired at $\mathrm{t}+\Delta \mathrm{t} \quad$ is $\mathrm{P}=\mu \Delta \mathrm{t}$ (the rate of repair, that is, the probability of reconstruction)[9].

According to the running state of the system, the state diagram shown in Fig.2 is obtained:

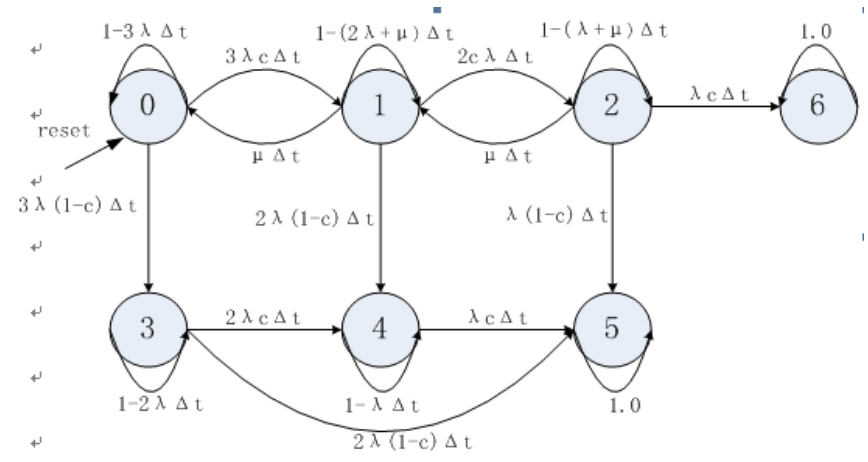

Figure 2. State diagram of reliability analysis for the three module system redundancy

A transfer density matrix, such as formula (4), is obtained based on the state diagram shown in Fig. 2:

$$
Q=\left[\begin{array}{ccccccc}
-3 \lambda & 3 c \lambda & 0 & 3(1-c) \lambda & 0 & 0 & 0 \\
\mu & -(2 \lambda+\mu) & 2 c \lambda & 0 & 2(1-c) \lambda & 0 & 0 \\
0 & \mu & -(\lambda+\mu) & 0 & 0 & (1-c) \lambda & c \lambda \\
0 & 0 & 0 & -2 \lambda & 2 c \lambda & 2(1-c) \lambda & 0 \\
0 & 0 & 0 & 0 & -\lambda & c \lambda & 0 \\
0 & 0 & 0 & 0 & 0 & 0 & 0 \\
0 & 0 & 0 & 0 & 0 & 0 & 0
\end{array}\right]
$$

$\mathrm{P}(\mathrm{t})$ can be obtained according to the formula $\frac{\mathrm{d}}{\mathrm{d}(\mathrm{t})} p(t)=p(t) Q$ and initial conditions $\mathrm{P}(0)$ $=[1,0,0,0,0,0]$. Thus, the probabilities of each state of the $t$ moment and the system reliability, $\mathrm{R}(\mathrm{t})$ $=\mathrm{p} 0(\mathrm{t})+\mathrm{p} 1(\mathrm{t})+\mathrm{p} 2(\mathrm{t})+\mathrm{p} 4(\mathrm{t})+\mathrm{p} 5(\mathrm{t})$ can be obtained[10]. Making $\lambda=0.0001, \mathrm{t},=1000, \mathrm{c}=0.95$, through the Matlab simulation, we can get the influence of different repair rate $\mu$ (the probability of reconfiguration rate) on the reliability.

Table 1 is the third mock redundant system reliability analysis results:

Table 1 The reliability analysis of the three module system redundancy

\begin{tabular}{|c|c|c|c|c|c|c|c|c|c|}
\hline$\mu$ & 0.0000 & 0.001 & 0.005 & 0.01 & 0.05 & 0.2500 & 0.9000 & 0.9900 & 1.0000 \\
\hline $\mathrm{R}(\mathrm{t})$ & 0.9862 & 0.9880 & 0.9888 & 0.9904 & 0.9937 & 0.9943 & 0.9948 & 0.9949 & 0.9951 \\
\hline
\end{tabular}

From the data of Table 1, the reliability of the system becomes stronger with the increase of reconfiguration probability. 


\section{Reliability Analysis of Four Modular Redundancy System}

The four module redundancy system similar to the three module redundancy system, are used in system reliability analysis of Markov process.

The operation of the system is divided into 6 states.

Status 0: four modules are normal, the output of the correct results. State 5: standby machine system two modules are failure, one can be detected, one can not be detected, the main subsystem of the two modules normal, the output of the correct results.

Fig. 3 shows the state diagram of the reliability analysis of the MIPS four modular redundant system.

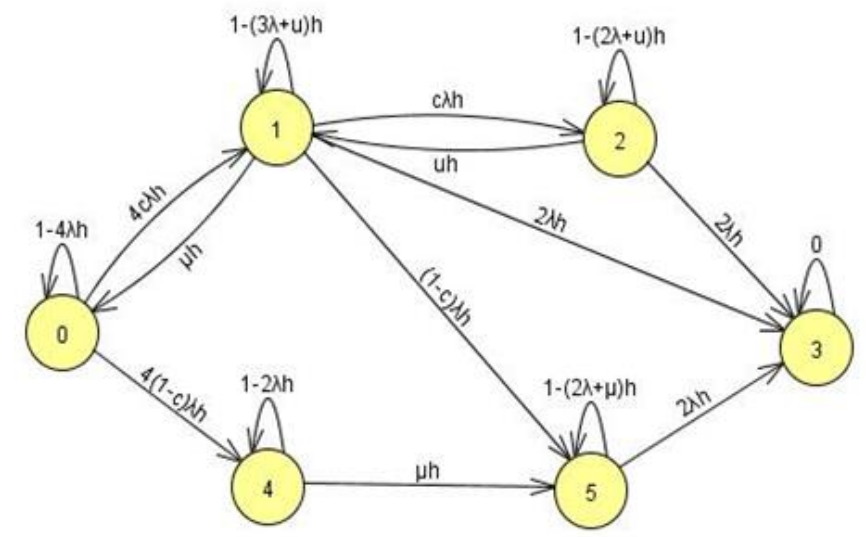

Figure 3. State diagram of reliability analysis for the four module system redundancy

The transfer density matrix is obtained according to the state diagram, such as formula (5):

$$
Q=\begin{array}{cccccc}
-4 l & 4 c l & 0 & 0 & 4(1-c) l & 0 \\
m & -(3 l+m) & c l & 2 l & 0 & (1-c) l \\
0 & m & -(2 l+m) & 2 l & 0 & 0 \\
0 & 0 & 0 & 0 & 0 & 0 \\
0 & 0 & 0 & 2 l & -2 l & 0 \\
0 & 0 & 0 & 2 l & m & -(2 l+m)
\end{array}
$$

$\mathrm{P}(\mathrm{t})$ can be obtained according to the initial condition $\mathrm{P}(0)=[1,0,0,0,0,0]$. System reliability $\mathrm{R}(\mathrm{t})$ $=\mathrm{p} 0(\mathrm{t})+\mathrm{p} 1(\mathrm{t})+\mathrm{p} 2(\mathrm{t})+\mathrm{p} 4(\mathrm{t})+\mathrm{p} 5(\mathrm{t})$ can be derived[10]. Making $\lambda=0.0001, \mathrm{t}=1000, \mathrm{c}=0.95$, through the Matlab simulation, we can obtain the influence of different reconstruction rate mu on reliability.

Table 2 The Reliability Analysis of The Four Module System Redundancy

\begin{tabular}{|c|c|c|c|c|c|c|c|c|c|}
\hline$\mu$ & 0.0000 & 0.0001 & 0.005 & 0.01 & 0.1 & 0.15 & 0.8000 & 0.9500 & 1.0000 \\
\hline $\mathrm{R}(\mathrm{t})$ & 0.9671 & 0.9680 & 0.9771 & 0.9817 & 0.9900 & 0.9954 & 0.9980 & 09986 & 0.9991 \\
\hline
\end{tabular}

From the data of Table 2, the reliability of the system becomes stronger with the increase of reconfiguration probability.

\section{Summary}

In this paper, multi-mode redundancy and control network reliability analysis for embedded system in the three module MIPS reconstruction and four mode MIPS reconstruction has done some 
exploratory work. Unlike other design, the design adopts dynamic reconfiguration method, which has many advantages, such as: to ensure the system automatic repair, debugging module of long-term residual, save chip space, improve chip utilization, ensure the system accuracy etc..

\section{Acknowledgements}

This paper is funded by sub subject of research project of science and technology of Jiangxi provincial education department in 2016. The original subject number is: GJJ161225. Sub project number is: GJKJ-16-17.

\section{References}

[1] Jinfeng Li, Huanle Zhu, Wenjie Zhang. Design of multi-modular redundancy system reliability based on the dynamic reconfiguration. ICEEECS2016, 2016.10.

[2] Jinfeng Li, Huanle Zhu, Wenjie Zhang. Application research on project driven in "electric information class" classroom teaching[J]. JOURNAL OF COMPUTERS, 2016.11.

[3] Jinfeng Li, Huanle Zhu, Wenjie Zhang. The application on EIP-CDIO cultivating model in the course of electronic electrical design classes[J]. CTMEE2016, 2016.06.

[4] A. David, P. John, L. Hennessy. Computer Organization and Design: The Hardware/Software Interface, Third Edition[M]. China Machine Press, 2012.

[5] X. Iturbe, K. Benkrid, A. Ebrahim, et al. Snake: An Efficient Strategy for the Reuse of Circuitry and Partial Computation Results in High-Performance Reconfigurable Computing[C]. 2012.

[6] D. E. Lenoski. Models of multiprocessor computing[C], Innovative Architecture for Future Generation High-Performance Processors and Systems, 2016.10:28-29.

[7] T. Dorta, J. Jimenez, J. L. Martin, et al. Overview of FPGA-Based Multiprocessor Systems[C]. International Conference on Reconfigurable Computing and FPGAs, 2014.12:273-278.

[8] M. H. Lee, H. Singh, G. Lu, et al. Design and Implementation of the Morphosys Reconfigurable Computing Processor[J]. Journal of VLSI Signal Processing Systems, 2013, 24(5): 164-172.

[9] M. A. Iqbal, U. S. Awan. An Efficient Configuration Unit Design for VLIW Based Reconfigurable Processors[C], IEEE International Conference on Multitopic, 2014.12:47-52.

[10] D. Harris, S. Harris. Digital Design and Computer Architecture[M]. Morgan Kaufmann, March 16, 2007. 